\title{
Isokinetic strength testing does not predict hamstring injury in Australian Rules footballers
}

\author{
K Bennell, H Wajswelner, P Lew, A Schall-Riaucour, S Leslie, D Plant, J Cirone
}

\begin{abstract}
Objective-To determine the relation of hamstring and quadriceps muscle strength and imbalance to hamstring injury using a prospective observational cohort study Method-A total of 102 senior male Australian Rules footballers aged 22.2 (3.6) years were tested at the start of a football season. Maximum voluntary concentric and eccentric torque of the hamstring and quadriceps muscles of both legs was assessed using a Kin-Com isokinetic dynamometer at angular velocities of 60 and 180 degrees/second. Twelve $(\mathbf{1 1 . 8 \%})$ players sustained clinically diagnosed hamstring strains which caused them to miss one or more matches over the ensuing season.

Results-There were no significant differences for any of the isokinetic variables comparing the injured and non-injured legs in players with unilateral hamstring strains $(n=9)$. Neither the injured nor the non-injured leg of injured players differed from the mean of left and right legs in non-injured players for any isokinetic variable. The hamstring to opposite hamstring ratios also did not differ between injured and non-injured players. A hamstring to opposite hamstring ratio of less than 0.90 and a hamstring to quadriceps ratio of less than 0.60 were not associated with an increased risk of hamstring injury. A significantly greater percentage of players who sustained a hamstring strain reported a history of hamstring strain compared with non-injured players $(p=$ 0.02 ). However, this was not related to muscle weakness or imbalance.
\end{abstract}

Conclusions-Isokinetic muscle strength testing was not able to directly discriminate Australian Rules football players at risk for a hamstring injury.

(Br F Sports Med 1998;32:309-314)

Keywords: hamstring strain; isokinetic dynamometry; concentric; eccentric; injury; football

Australian Rules football is a popular game in Australia. The game is contested between two teams of 18 players on a large oval (up to 185 $m$ end to end), and at the senior level it consists of four quarters of 25 minutes each. The basic skills required include sprinting over short distances, tackling, jumping to mark high balls, and kicking and handballing the oval shaped ball a range of distances $(10 \mathrm{~m}$ up to $60 \mathrm{~m}$ ).

Hamstring strains are common in Australian Rules football as well as other sports involving sprinting and jumping. ${ }^{12}$ In elite level football, they are the most frequent injuries (86.4 injuries per 10000 playing hours) and account for the most time missed. ${ }^{1}$ Recurrence rates for hamstring strains also tend to be high despite concentrated rehabilitation efforts. Indeed, $34 \%$ of hamstring strains in professional footballers are recurrences. Given the personal and financial costs associated with such injuries, it is important to identify individuals at risk for hamstring strains to facilitate the implementation of preventive strategies.

During running, the hamstring muscles become active in the last third of the swing phase undergoing eccentric contraction to decelerate knee extension and oppose the activity of the quadriceps. At ground contact, the hamstrings switch from maximal eccentric to concentric activity and develop the greatest force of any lower extremity muscles. ${ }^{3}$ During kicking, the hamstrings are lengthened across both the hip and the knee. Thus the hamstring muscles are subjected to high forces during both open and closed kinetic chain activities making them vulnerable to injury.

Given the function of the hamstrings during sprinting and kicking, it is feasible that reduced strength and/or endurance of the hamstring muscles, inequality of the strength of left and right hamstring muscles or strength imbalances between quadriceps and hamstring muscles may predispose to hamstring strain. However, there have been few prospective studies in large athletic populations of the relation between these risk factors and the subsequent occurrence of hamstring injury. Furthermore, in many cases, the clinicians making the diagnosis of hamstring injury were not blinded to the results of the strength tests thus introducing the potential for bias.

The literature is conflicting about the relation between muscle strength and hamstring injury. Several early studies considered that a greater than $10 \%$ bilateral deficit in isometric hamstring strength was predictive of hamstring injury, ${ }^{45}$ and that reductions in hamstring strains could be achieved by implementing a programme to correct strength deficits and improve flexibility. ${ }^{6}$ These findings have been supported by prospective studies in 64 collegiate track and field athletes, of whom 26 sustained a hamstring injury during a two year follow up, ${ }^{7}$ and in 37 professional Australian Rules footballers, six of whom sustained hamstring injuries during one season. ${ }^{8}$ In the latter study, hamstring injury was associated with significantly lower concentric isokinetic hamstring to quadriceps muscle peak torque ratio and a lower hamstring muscle side to side peak torque at 60 degrees/second.

Conversely, other prospective and retrospective data fail to support a relation between mus- 
cle weakness or imbalance and hamstring injury. Liemohn $^{9}$ prospectively found no differences in isometric hamstring/quadriceps ratios between hamstring injured and non-injured groups of track and field athletes. Retrospective studies have also reported no significant differences between hamstring-injured and control athletes in hamstring strength indices of isokinetic concentric and eccentric peak torques. ${ }^{10}{ }^{11}$ The inability of research to consistently show a significant relation between hamstring strength parameters and injury may be due to methodological differences (study design, sample size, type of athletes, and method of strength testing) or to the influence of confounding variables ${ }^{12}$ such as the potential for bias in unblinded clinicians. Further research using larger sample sizes is needed to clarify the role of muscle strength in the prediction of hamstring injury. Another area that requires investigation is whether the ratio of eccentric hamstrings to concentric quadriceps strength is related to hamstring injury. Aagaard et $a l^{13}$ stated that this ratio may serve as a good indicator of the capacity of the hamstring to provide dynamic stability in terminal knee extension. This facet of dynamic knee control may be important during the running, kicking, and twisting activities that form part of Australian Rules football. There have been no studies to date relating this ratio to the likelihood of hamstring strain.

The aim of our prospective study was to determine whether isokinetic concentric and eccentric strength testing of the quadriceps and hamstring muscles performed at the commencement of an Australian Rules football season can predict the subsequent occurrence of hamstring injury throughout the season. In addition to the usual muscle ratios, we evaluated the eccentric hamstring to concentric quadriceps strength ratio in relation to hamstring injury. The significance of this research is that findings will assist in establishing the appropriateness of screening procedures to identify players at risk for hamstring injury.

\section{Materials and methods}

STUDY DESIGN

A prospective cohort design was used in this study.

SUBJECTS

A total of 102 male Australian Rules footballers were recruited from six professional Australian Football League teams and four A grade amateur teams. The mean (SD) age of the subjects was 22.2 (3.6), the mean height was 185.0 $(6.7) \mathrm{cm}$ and the mean weight was $84.3(7.6)$ kg. Eighty nine $(87 \%)$ subjects reported that they were right leg dominant with respect to kicking and 13 (13\%) were left leg dominant. The project was approved by the Human Research Ethics Committee of the University of Melbourne and all subjects provided written informed consent.

PROCEDURE

All players were tested before the commencement of either the 1996 or 1997 football season. Subjects were requested not to train or exercise vigorously during the four hours preceding testing. A questionnaire was completed by each subject to establish lower limb musculoskeletal injury history particularly in relation to hamstring strains. All tests were performed using identical procedures consisting of a warm up, test, and cool down. Warm up and cool down comprised 10 minutes of stationary cycling followed by lower limb muscle stretching.

Maximum voluntary concentric and eccentric torque of the hamstring and quadriceps muscles of both legs was assessed using a KinCom AP isokinetic dynamometer (Chattecx Corporation, Chattanooga, Tennessee, USA) at angular velocities of 60 and 180 degrees/ second through a range of $5-95^{\circ}$ of knee flexion and extension. All torques were corrected for the effects of gravity on the lower leg segment and the resistance pad of the dynamometer at $30^{\circ}$ knee flexion.

For isokinetic testing, subjects were positioned sitting upright and secured using torso, pelvic, thigh, and shin stabilisation straps. A back pad was used if necessary to establish a trunk angle of $90^{\circ}$. The input shaft of the dynamometer was aligned with the axis of rotation of the subject's knee considered to be the point at the centre of a line that passes transversely through the femoral condyles. The shin pad attachment was placed 1 to $2 \mathrm{~cm}$ proximal to the subject's lateral malleolus. A standardised testing protocol was followed for each subject with consistent verbal instructions given. The order of leg testing was randomised. Testing was first performed at 60 degrees/ second, and followed by 180 degrees/second. At each speed, the quadriceps muscles were tested first followed by the hamstrings. Concentric strength was assessed before eccentric strength. Subjects performed a submaximal warm up on the Kin-Com before testing to familiarise themselves with the protocol. This was then followed by five or six maximal contractions for data collection with a rest interval of about 60 seconds between each.

Neither players nor their respective medical staff were given the results of the tests, and no specific rehabilitation programmes were implemented on the basis of strength deficits detected from testing. This was to ensure that we were testing the predictive value of preseason screening.

DIAGNOSIS OF HAMSTRING STRAIN

Players were monitored throughout the 1996 or 1997 football season. Injury diagnosis was made by the medical staff at each of the participating clubs. A hamstring muscle strain was diagnosed clinically and included in the study if all the following criteria were met: $(a)$ sudden onset of pain in the hamstring muscle during a match or training; (b) pain in the hamstring muscle with contraction of the muscle and with stretching; (c) tenderness to palpation; (d) an injury severe enough to cause the player to miss an official match. The medical staff were encouraged to obtain a diagnostic ultrasound examination within the first week of 
injury to confirm the presence of a hamstring muscle lesion.

\section{STATISTICAL ANALYSIS}

For each test velocity and each test movement the maximal torque value for concentric and eccentric muscle activity was used in data analysis. The parameters of interest were absolute maximum torque, relative maximum torque (maximal torque per body weight), concentric and eccentric hamstring to quadriceps ratios, eccentric hamstring to concentric quadriceps ratios, and hamstring to opposite hamstring ratios. Paired $t$ tests were used to compare $(a)$ the non-dominant and dominant leg, (b) the injured and non-injured legs in injured players, and $(c)$ legs with and without a history of hamstring strain. Independent $t$ tests were used to compare $(a)$ the non-injured leg of the injured players with the mean of left and right legs for the non-injured group and (b) hamstring to opposite hamstring ratio in the injured and non-injured players.

The ability of preseason Kin-Com testing to predict hamstring injury was evaluated using the $\chi^{2}$ test. The strength test variables were converted to dichotomous variables consisting of (a) players with and without hamstring to opposite hamstring ratios of less than 0.90 on either leg and $(b)$ players with and without hamstring to quadriceps ratios of less than 0.60 on either leg.

Differences in a past history of hamstring injury between injured and non-injured players were analysed using the $\chi^{2}$ test. A significance level of $\mathrm{p}<0.05$ was set for all tests.

\section{Results}

INCIDENCE OF HAMSTRING STRAINS

Twelve of the 102 players $(11.8 \%)$ sustained one or more clinically diagnosed hamstring muscle strains which caused them to miss playing time during the study. Two players sustained a hamstring muscle strain on each leg during the season. Eight $(57 \%)$ of the 14 muscle injuries were confirmed by ultrasound examination. Nine $(64 \%)$ of the injuries were on the right leg and five $(36 \%)$ were on the left. This represented 10 $(71 \%)$ on the dominant (kicking) leg and four $(29 \%)$ on the non-dominant leg. The number of matches missed because of the injury (with matches about one week apart) ranged from one to seven with a mean of 2.8 (1.7).

\section{COMPARISON OF DOMINANT VERSUS}

NON-DOMINANT LEG

There was no significant difference between dominant and non-dominant legs, defined with respect to kicking, for any of the isokinetic variables in the group as a whole and in the injured and non-injured players separately $(p>0.05)$. Therefore further analyses were performed without regard for leg dominance.

COMPARISON OF INJURED VERSUS NON-INJURED LEGS IN INJURED PLAYERS

Table 1 gives mean (SD) values for the absolute hamstring and quadriceps concentric and eccentric muscle strength variables for injured
Table 1 Mean (SD) absolute peak torque and ratios for injured and non-injured legs of footballers who sustained hamstring injuries

\begin{tabular}{lrrr}
\hline Variable & \multicolumn{1}{l}{$\begin{array}{l}\text { Injured leg } \\
(n=9)\end{array}$} & \multicolumn{2}{c}{$\begin{array}{l}\text { Non-injured } \\
\text { leg }(n=9)\end{array}$} \\
\hline Quad Con 60 (N.m) & $226.5(53.6)$ & $264.3 \quad(52.5)$ \\
Quad Ecc 60 (N.m) & $315.2(74.8)$ & $341.0(76.2)$ \\
Ham Con 60 (N.m) & $154.3(25.0)$ & $154.0(33.6)$ \\
Ham Ecc 60 (N.m) & $189.5(33.1)$ & $181.1(28.7)$ \\
Quad Con 180 (N.m) & $215.8(41.7)$ & $225.3(41.2)$ \\
Quad Ecc 180 (N.m) & $340.0(78.7)$ & $339.9(78.7)$ \\
Ham Con 180 (N.m) & $154.8(30.4)$ & $158.8(42.0)$ \\
Ham Ecc 180 (N.m) & $188.2(28.3)$ & $180.5(31.0)$ \\
Ham/Quad Con 60 & $0.69(0.19)$ & $0.59(0.12)$ \\
Ham/Quad Ecc 60 & $0.60(0.11)$ & $0.55(0.11)$ \\
Ham/Quad Con 180 & $0.73(0.11)$ & $0.73(0.19)$ \\
Ham/Quad Ecc 180 & $0.57(0.10)$ & $0.56(0.08)$ \\
HamEcc/QuadCon 60 & $0.83(0.16)$ & $0.70(0.14)$ \\
HamEcc/QuadCon 180 & $0.88(0.10)$ & $0.83(0.11)$ \\
\hline
\end{tabular}

Quad, quadriceps muscle; Ham, hamstring muscle; Con, concentric torque; Ecc, eccentric torque.

and non-injured legs in injured players. Two players could not be included as they sustained a hamstring strain on both legs during the study. Results of paired $t$ tests showed no significant differences between legs for any of the variables $(p>0.05)$.

COMPARISON OF INJURED PLAYERS WITH NON-INJURED PLAYERS

Table 2 gives mean (SD) values for the relative hamstring and quadriceps concentric and eccentric muscle strength variables for the noninjured leg in injured players and the mean of left and right legs in non-injured players. For those players who injured both hamstrings, the mean of the legs was used in analysis. Independent $t$ tests showed no significant differences between the injured and non-injured players for any of the variables. There was also no significant difference between the injured leg of the injured players and the mean of left and right legs in non-injured players.

Table 2 Mean (SD) relative peak torque and ratios for the non-injured leg of hamstring injured plavers and the mean of left and right legs in non-injured footballers

\begin{tabular}{lll}
\hline & $\begin{array}{l}\text { Injured } \\
\text { players } \\
(n=12)\end{array}$ & $\begin{array}{l}\text { Non-injured } \\
\text { players } \\
(n=90)\end{array}$ \\
\hline Quariable & $3.0(0.6)$ & $2.8(0.4)$ \\
Quad Ecc 60 (N.m/kgBW) & $3.9(0.9)$ & $4.0(0.7)$ \\
Ham Con 60 (N.m/kgBW) & $1.8(0.3)$ & $1.7(0.3)$ \\
Ham Ecc 60 (N.m/kgBW) & $2.1(0.3)$ & $2.1(0.4)$ \\
Quad Con $180(\mathrm{~N} . \mathrm{m} / \mathrm{kgBW})$ & $2.6(0.4)$ & $2.4(0.3)$ \\
Quad Ecc $180(\mathrm{~N} \cdot \mathrm{m} / \mathrm{kgBW})$ & $3.9(0.8)$ & $4.0(0.7)$ \\
Ham Con $180(\mathrm{~N} \cdot \mathrm{m} / \mathrm{kgBW})$ & $2.6(0.4)$ & $2.4(0.3)$ \\
Ham Ecc $180(\mathrm{~N} . \mathrm{m} / \mathrm{kgBW})$ & $2.1(0.3)$ & $2.2(0.4)$ \\
Ham/Quad Con 60 & $0.59(0.12)$ & $0.59(0.10)$ \\
Ham/Quad Ecc 60 & $0.55(0.11)$ & $0.53(0.12)$ \\
Ham/Quad Con 180 & $0.73(0.19)$ & $0.67(0.15)$ \\
Ham/Quad Ecc 180 & $0.56(0.08)$ & $0.56(0.17)$ \\
HamEcc/QuadCon 60 & $0.70(0.14)$ & $0.74(0.16)$ \\
HamEcc/QuadCon 180 & $0.83(0.11)$ & $0.90(0.23)$ \\
\hline
\end{tabular}

Quad, quadriceps muscle; Ham, hamstring muscle; Con, concentric torque; Ecc, eccentric torque; BW, body weight.

Table 3 Mean (SD) of hamstring to opposite hamstring muscle ratio comparing the injured and non-injured players

\begin{tabular}{lll}
\hline Variable & $\begin{array}{l}\text { Injured players } \\
(n=12)\end{array}$ & $\begin{array}{l}\text { Non-injured } \\
\text { players }(n=90)\end{array}$ \\
\hline Ham:opp Ham 60 Con & $0.99(0.15)$ & $1.04(0.16)$ \\
Ham:opp Ham 60 Ecc & $0.83(0.13)$ & $0.83(0.19)$ \\
Ham:opp Ham 180 Con & $1.05(0.19)$ & $1.02(0.27)$ \\
Ham:opp Ham 180 Ecc & $1.22(0.22)$ & $1.28(0.30)$ \\
\hline
\end{tabular}

*Right hamstring : left hamstring.

Ham, hamstring; Con, concentric; Ecc, eccentric. 
Table 3 gives the mean (SD) hamstring to opposite hamstring ratio at both speeds for the injured and non-injured players. There was no significant difference between the two groups in terms of side to side hamstring muscle balance.

PREDICTION OF HAMSTRING INJURY

A hamstring to opposite side hamstring ratio of less than 0.90 was found for $47 \%$ of players at concentric 60 degrees/second, $63 \%$ at eccentric 60 degrees/second, $41 \%$ at concentric 180 degrees/second, and $60 \%$ at eccentric 180 degrees/second. However, the risk of subsequent hamstring strain did not differ for those with and without ratios less than 0.90 except for eccentric 180 degrees/second where those with ratios greater than 0.90 actually were at higher risk $(\mathrm{p}=0.02)$.

Hamstring to quadriceps ratios of less than 0.60 were also common in the players tested. For example, $77 \%$ had a ratio of less than 0.60 at concentric 60 degrees/second, $35 \%$ at concentric 180 degrees/second, $86 \%$ at eccentric 60 degrees/second, and $82 \%$ at eccentric 180 degrees/second. The risk of hamstring injury could not be predicted by these ratios $(p>0.05)$.

PAST HISTORY OF HAMSTRING STRAIN

Thirty five players reported a past history of hamstring injury on one or both legs. There was no significant difference for any of the left leg absolute or relative muscle strength variables comparing those with and without a history of left hamstring strain $(p>0.05)$. However, those with a history of right hamstring strain had significantly greater right leg absolute and relative concentric hamstring muscle strength at both 60 degrees/second (absolute: $p$ $=0.001$; relative: $\mathrm{p}=0.02)$ and at 180 degrees $/$ second (absolute: $\mathrm{p}=0.002$; relative: $\mathrm{p}=0.01$ ) than those without a history of right hamstring strain. At both testing speeds, the difference was about $10 \%$ for absolute values and $15 \%$ for relative values.

A significantly greater percentage of players who sustained a hamstring injury during the study reported a history of hamstring injury compared with the non-injured group (66\% vs $31 \% ; p=0.02)$. Football players with a past history of hamstring injury were 2.1 times more likely to sustain another hamstring injury than those with no past history.

\section{Discussion}

We used isokinetic dynamometry to assess concentric and eccentric muscle strength and muscle imbalance of the hamstring and quadriceps at two different speeds of movement in 102 male Australian Rules footballers. The results of our prospective cohort study do not support an association between preseason muscle weakness or imbalance and subsequent occurrence of hamstring muscle strain. There was no significant difference between injured and non-injured legs in those players who sustained a unilateral injury while the relative strength of the non-injured leg of injured players was similar to that of non-injured players.
Furthermore, imbalances in side to side hamstring strength of more than $10 \%$ or hamstring to quadriceps ratios of less than $60 \%$ on either leg did not place a player at greater risk for subsequent hamstring injury. These results imply that isokinetic muscle strength testing performed at the commencement of a football season is unlikely to predict the risk of hamstring injury during the season.

We did not corroborate the concentric findings of a similar but smaller study in Australian Rules footballers. ${ }^{8}$ There are several reasons for this disparity. Firstly, we sampled a range of professional and high level amateur players whereas the 37 subjects in the Orchard study were all elite professional footballers from one particular team. It is possible that their results do not generalise to footballers from other teams and competitions. For example, they suggested that players are at a substantially greater risk of hamstring muscle strain when they have hamstring to quadriceps muscle ratios (at 60 degrees/second) of less than 0.61 . However, the mean ratio for both injured and non-injured players in our cohort was 0.59 and over $76 \%$ of players had ratios of less than 0.60 . This could indicate that the two cohorts are not directly comparable. Secondly, we specifically excluded all players with back related hamstring injuries, and, although not all players underwent diagnostic ultrasound testing, we are confident that our injured players sustained isolated hamstring muscle injuries. The addition of a back related component to the injury in some of their subjects may have influenced the results. Thirdly, the diagnosis of hamstring injury in their study was made in an unblinded fashion with the clinician privy to the results of the isokinetic testing. Thus the potential for bias cannot be entirely excluded. Finally, we analysed our data in a different manner to avoid treating each leg as an independent data point which is not strictly correct.

Our findings agree with others who have failed to establish a significant relation between strength testing and hamstring muscle injury. ${ }^{9-11}$ It may be argued that a relation was missed by failing to measure peak torque at faster speeds, such as 300 degrees/second, which are closer to the speed of muscle contraction during sporting activity. ${ }^{14}$ However, associations between preseason muscle weakness and hamstring muscle injury have been reported using slower testing speeds of 60 degrees/second ${ }^{8}$ as this speed may accentuate the presence of ratio deficits.

Although no predictive relation was found between preseason isokinetic muscle screening and hamstring injury, our results do not necessarily exclude muscle weakness or imbalance as playing a role. Because of the time lag between testing and injury, intervening factors may have altered a player's strength profile during the season thereby rendering the preseason results less useful for injury prediction. Attempts were made to test players after their weight training module when maximal strength gains would have already occurred. However, it is possible that fluctuations in muscle strength occurred during the season depending on other injuries or training protocols. Extensive monitoring of 
players to determine whether this may have been the case was not available. Although serial isokinetic testing throughout the season may be more useful in detecting players at risk of hamstring injury, this may not be possible from a practical perspective.

Isokinetic dynamometry as used in our study provides an indication of muscle strength in an open kinetic chain activity. However, in Australian Rules football, hamstring injury appears anecdotally to be more related to running, a closed kinetic chain activity, rather than kicking. It may be that functional tests such as the single leg hop provide a better indication of the function of the hamstring muscles and thus injury risk. Low to moderate correlations have been found between isokinetic and functional tests, ${ }^{15}$ which may indicate that open and closed kinetic chain measurements do provide different information about muscle performance. The role of functional tests in predicting hamstring strain warrants investigation.

The cause of hamstring strain is probably multifactorial and it is not surprising that measurement of a single factor fails adequately to predict their occurrence. Other factors including muscle flexibility, ${ }^{9}$ degree of lumbar lordosis, ${ }^{16}$ and neural tension ${ }^{17}$ have all been suggested as possible predisposing factors. Furthermore, different risk factors may be operating in different sports and in different individuals, making it difficult to establish predictive relations from large athlete cohorts.

Our results did show that players with a history of hamstring strain were more at risk of sustaining a subsequent strain than those without. This could either suggest that predisposing factors were still operating in these players or that rehabilitation from the previous hamstring strain was inadequate. However, the association between injury history and subsequent strain was independent of muscle weakness or imbalance. In fact, those with a previous right hamstring strain actually had significantly higher absolute and relative right hamstring strength than players with no history of hamstring strain, suggesting that the strengthening programmes during rehabilitation were indeed effective. This tends to suggest that strength per se is unrelated to hamstring strain and that other factors are more important. Whatever the causal mechanism, a history of hamstring strain can be used as a marker to identify at risk individuals.

Since players with previous hamstring strains were 2.1 times more likely to experience another strain during the time course of the study and these players showed greater concentric hamstring strength than players without previous injury, it might be expected that the players who were injured during the study would show greater concentric strength than those players not exposed to injury. However, this was not shown to be the case. The reason for this is probably that greater concentric strength was only found in those with injury to the right hamstring and not the left, thus reducing the likelihood of this result reaching statistical significance.
The concentric hamstring to quadriceps strength ratios found in our study $(0.69-0.73$ and $0.59-0.73$ ) seem somewhat higher than those previously reported in the literature based on gravity corrected isokinetic peak torques obtained in well trained subjects $(0.44-0.69) .{ }^{8} 13$ In contrast, the eccentric ratios are comparable with those in previous reports. Although the exact reasons for this disparity are unclear, it is possible that Australian Rules footballers have very strong hamstrings when contracting concentrically. This may be due to weight training programmes that emphasise hamstring strengthening in an attempt to prevent the high incidence of hamstring strains that occur in this sport.

The hamstring muscle strain incidence rate in our study, $11.8 \%$, is relatively high. It could be argued that, as the cohort was not randomly selected, players most at risk for this injury volunteered to participate. However, the number sustaining a hamstring strain is similar to the rates described in previous studies of football injuries at this level: $8 \%$ at the amateur level ${ }^{18}$ and $14 \%$ at the elite level. ${ }^{1}$ The frequency of this injury in Australian Rules football highlights the need for further research aimed at identifying risk factors and the most effective preventive strategies.

In conclusion, as isokinetic strength testing was unable to discriminate between injured and uninjured players or predict the likelihood of injury, its role as a preseason screening tool to identify footballers at risk for hamstring strain may be limited. Since a past history of hamstring strain is associated with a significantly greater risk for subsequent strain, preventive programmes should be particularly directed toward these players.

This project was funded by a grant from the Australian Physiotherapy Association (Victorian Branch). We would like to thank the players and clubs who participated, in particular: Loris Bertolacci and Bruce Connor (Essendon Football Club), Sallie Cowan and Rebecca Bale (Uni Blues Football Club), Brendan Curry (Williamstown Football Club), Andrew MitchBrendan Curry (Williamstown Football Club), Andrew Mitchwood Football Club), Mark Smythe and Simon McCauley (Footscray Football Club), Peter Thomas (St Kilda Football Club), Lachlan Kellaway (Sandringham Football Club), Mike McCoy (Richmond Football Club), David Butterford (North Melbourne Football Club).

Kim Bennell helped to design the protocol, recruit the subjects, and organise the data collection, assisted with the data analysis, and wrote the paper. Henry Wajswelner helped to formulate the study hypothesis, designed the protocol, and assisted in data collection. Paul Lew initiated the research, discussed core ideas, participated in protocol design and subject recruitment, and edited the paper. Andreas Schall-Riaucour, Sam Leslie, and David Plant assisted in data collection and subject recruitment. Jason Cirone participated in data analysis, interpretation of the findings, and writing of the paper.

1 Seward H, Orchard J, Hazard H, et al. Football injuries in Australia at the elite level. Med $\mathcal{F}$ Aust 1993;159:298-301.

2 Bennell KL, Crossley K. Musculoskeletal injuries in track and field: incidence, distribution and risk factors. Aust f Sci Med Sport 1996;28:69-75.

3 Mann RA, Moran GT, Dougherty SE. Comparative electromyography of the lower extremity in jogging, running, and sprinting. Am f Sports Med 1986;14:501-10.

4 Burkett L. Causative factors of hamstring strains. Med Sci Sports Exerc 1970;2:39-42.

5 Christensen C, Wiseman D. Strength, the common variable in hamstring strain. Athletic Training 1972;7:36-40.

6 Heiser TM, Weber J, Sullivan G, et al. Prophylaxis and management of hamstring muscle injuries in intercollegiate football players. Am $\mathcal{F}$ Sports Med 1984;12:368-70.

7 Yamamoto T. Relationship between hamstring strains and leg muscle strength. F Sports Med Phys Fitness 1993;33:194-9.

8 Orchard J, Marsden J, Lord S, et al. Preseason hamstring muscle weakness associated with hamstring muscle injury in Australian footballers. Am $\mathcal{F}$ Sports Med 1997;25:81-5. 
Liemohn W. Factors related to hamstring strains. 7 Sports Med 1978;18:71-6.

10 Worrell TW, Perrin DH, Gansneder B, et al. Comparison of isokinetic strength and flexibility measures between hamstring injured and noninjured athletes. F Orthop Sports Phys Ther 1991;13:118-25.

11 Paton RW, Grimshaw P, McGregor J, et al. Assessment of the effects of significant hamstring injury: an isokinetic study. F Biomed Eng 1989;11:229-30.

12 Worrell TW, Perrin DH. Hamstring muscle injury: the influence of strength, flexibility, warm-up, and fatigue. $\mathcal{F}$ Orthop Sports Phys Ther 1992;16:12-18.

13 Aagaard P, Simonsen EB, Trolle M, et al. Isokinetic hamstrings quadriceps strength ratio: influence from join angular velocity, gravity correction and contraction mode. Acta Physiol Scand 1995;154:421-7.
14 Knapik JJ, Bauman CL, Jones BH, et al. Preseason strength and flexibility imbalances associated with athletic injuries in female collegiate athletes. Am F Sports Med 1991;19:76-81.

15 Pincivero DM, Lephart SM, Karunakara RG. Relation between open and closed kinematic chain assessment of knee strength and functional performance. Clin $\mathcal{F}$ Sport Med 1997;7:11-16.

16 Hennessy L, Watson AWS. Flexibility and posture assessment in relation to hamstring injury. Br $\mathcal{F}$ Sports Med 1993; 27:243-6.

17 Kornberg C, Lew P. The effect of stretching neural structures on grade one hamstring injuries. F Orthop Sports Phys Ther 1989;10:481-7.

18 Shawdon A, Brukner P. Injury profile of amateur Australian Rules footballers. Aust F Sci Med Sport 1994;26:59-61.

\section{Commentary}

My compliments to the authors for this very well written and well conducted study. One of the greatest challenges to the sports medicine practitioner is, perhaps, accepting an assumption that a pre-existing strength deficit is present in an injured athlete. The work of these authors not only addresses the predictive value of isokinetic dynamometry, but it also highlights a much more significant issue: prospective cohort design studies. With respect to this issue, the lack of studies using this particular research design is a contributing factor to the many "inconclusive findings" that exist within the literature. For example, the anterior cruciate ligament (ACL) injured individual has been the focus of countless studies, and thus far, only general agreement has been reached about the use of closed kinetic chain exercises. It is interesting to note that this agreement has been based, in large part, on a popular and well detailed review paper. ${ }^{1}$

Although I would acknowledge that conducting prospective cohort studies is a challenging task, it is none the less a necessary avenue for future sports medicine research. The scientific literature has too often relied on conclusions drawn from strength evaluations, functional outcome surveys, balance, functional performance, and neuromuscular "profiles" of ACL injured individuals. The time has now come to apply the sophistication of various anatomical and physiological measurement techniques to answering time mediated research questions. Once again, the authors must be commended for their efforts and for bringing to light the appropriateness of their prospective design relative to their research question.

D C PINCIVERO 